\title{
INTERSUBBAND COLLECTIVE MODES IN MULTIWIRE SUPERLATTICES*
}

\author{
M. ZALUŻNY \\ Institute of Physics, M. Curie-Sklodowska University \\ pl. M. Curie-Sklodowskiej 1, 20-031 Lublin, Poland
}

Intersubband plasma excitation spectrum of lateral multiwire superlatlices is investigated theoretically within the random-phase approximation. We examine the role of an interwire electron-electron interaction in regard to the depolarization shift.

PACS numbers: 78.65.Fa, 73.20.Mf, 78.30.Fs

In recent years it has been demonstrated expcrimentally that it is possible to fabricate lateral multiwire superlattices (LMWSL's) consisting of quantum wires a few hundred angstrom wide. Raman spectroscopy $[1,2]$ and far-infrared al ... orption $[\lambda, 4]$ experiments are the two main experimental techniques to study collective excitations in such quasi-one-dimensional electron systems. This note is an extension of the theory of the intersubband transitions (in LMWSL's) developed in Refs. $[5,6]$.

We concentrate on an estimate of the relative contribution of the interwire and intrawire e-e interaction to the depolarization shift of the intersubband transitions in LMWSL's. In contrast to the previous papers, where the intersubband transitions induced by the light with polarization $\boldsymbol{e}=(0,1,0)$ were only considered, we discuss also the case when $e=(0,0,1)$. (We assume here that: (i) wires lie in the plane $z=0$ and are parallel to the $x$-axis and (ii) there is no overlap between neighbouring wave functions.)

The one-electron wave function of the system can be written in the tight-binding form

$$
\left|k_{x}, k_{y}, n_{y}, n_{z}\right\rangle=\exp \left(\mathrm{i} k_{x} x\right) \frac{1}{\sqrt{N}} \sum_{n} \exp \left(\mathrm{i} k_{y} n d\right) \phi_{n_{y}}(y-n d) \chi_{n_{z}}(z),
$$

where $n_{y}, n_{z}$ label the subbands connected with quantization in the $y$-direction and $z$-direction, respectively, $n$ labels the wire, $N$ is the total number of the wires, $d$ is a period of the superlattice, $k_{y}=2 \pi p / N d\left(-\pi / d<k_{y}<\pi / d\right)$ is the component of the superlattice wave vector in the first Brillouin zone where $p$ is an integer, $\chi_{n_{z}}(z)$ and $\phi_{n_{y}}(y)$ are the wave functions (of the isolated wire) in the $z$-direction and the $y$-direction, respectively.

*This work is supported by the Committee for Scientific Research under contract No. 203109101. 
The eigenenergy of the system is

$$
\mathcal{E}\left(k_{x}, n_{y}, n_{z}\right)=\hbar^{2} k_{x}^{2} / 2 m+E_{n_{y} n_{z}}
$$

$m$ is the effective mass.

In the further calculations we restrict ourselves to the simplest case where there are only two subbands in each direction $\left(n_{y}, n_{z}=0,1\right)$. We assume also that only the ground subband $n_{y}=n_{z}=0$ is occupied.

Following the self-consistent field (SCF) scheme of Ehrenreich and Cohen [7] introduced in [5], we find after a lengthy manipulation (details will be published elsewhere) the dispersion relation for two coupled intersubband modes $\tilde{\omega}_{10}$ and $\tilde{\omega}_{01}$. In the long-wavelength limit $(q \rightarrow 0)$ the coupling between the modes can be neglected and their frequencies are given by

$$
\tilde{\omega}_{\alpha \alpha^{\prime}}^{2}=\tilde{\omega}_{\alpha \alpha^{\prime}}^{2}+\frac{4 \pi N_{1} e^{2} \omega_{\alpha \alpha^{\prime}}}{\varepsilon} g_{\alpha \alpha^{\prime}} \quad(q \rightarrow 0), \quad \alpha, \alpha^{\prime}=0,1,
$$

where $\hbar \omega_{\alpha \alpha^{\prime}}=E_{\alpha \alpha^{\prime}}-E_{00}, N_{\mathrm{l}}$ is the linear density of electrons and $\varepsilon$ is the background dielectric constant. The function $g_{\alpha \alpha^{\prime}}(q)$ is given by

$$
g_{\alpha \alpha^{\prime}}(q)=\sum_{m} F_{\alpha}\left(x_{m}\right) I_{\alpha^{\prime}}\left(x_{m}\right) / x_{m},
$$

where

$$
\begin{aligned}
& F_{\alpha}\left(x_{m}\right)=\int \mathrm{d} z \mathrm{~d} z^{\prime} \exp \left(-x_{m}\left|z-z^{\prime}\right|\right)\left|\chi_{0}(z)\right|^{2}\left|\chi_{\alpha}\left(z^{\prime}\right)\right|^{2}, \\
& I_{\alpha}\left(x_{m}\right)=\left|\int \mathrm{d} y \exp \left(-x_{m} y\right) \phi_{0}^{*}(y) \phi_{\alpha}(y)\right|^{2},
\end{aligned}
$$

and $x_{m}=q+2 \pi m / d$.

The equation for $\tilde{\omega}_{10}$ is identical with the expression derived by Que et al. [5], while the equation for $\tilde{\omega}_{01}$ is derived here for the first time. The mode with the frequency $\tilde{\omega}_{\alpha \alpha^{\prime}}$ is connected with $\left|k_{x}, k_{y}, 0,0\right\rangle \rightarrow\left|k_{x}, k_{y}, \alpha, \alpha^{\prime}\right\rangle$ transitions and can be excited by the light with polarization $e=(0,1,0)$, for $\alpha=1, \alpha^{\prime}=0$ and polarization $e=(0,0,1)$ for $\alpha=0, \alpha^{\prime}=1$.

From Eq. (3) we find that energies of collective excitations $\hbar \tilde{\omega}_{\alpha \alpha^{\prime}}$ are shifted from single-particle excitation energies $\hbar \tilde{\omega}_{\alpha \alpha^{\prime}}$. The shift is caused by Coulomb's interaction between electrons, which exists between electrons in different quantum wires as well as those in the same wire. In the long-wave length limit the strength of the Coulomb interaction for mode $\alpha \alpha^{\prime}$ is determined by the factor $g_{\alpha \alpha^{\prime}} \equiv$ $g_{\alpha \alpha^{\prime}}(q \rightarrow 0)$. We have calculated $g_{10}$ and $g_{01}$ assuming that the effective potential for electrons (in the $z$ - and $y$-directions) has a square well form

$$
V(y, z)= \begin{cases}0 & \text { for }|y|<a_{y} / 2 \text { and }|z|<a_{z} / 2, \\ \infty & \text { otherwise. }\end{cases}
$$

The inclusion of more realistic quantizing potential is straightforward but this is sufficient to estimate how strongly the interwire interaction modifies the depolarization shift. Moreover, for the confinement given by Eq. (7) the integrations appearing in the definitions (5) and (6) can be performed analytically. Because of space limitation we restrict ourselves to the presentation of the final results for $g_{\alpha \alpha^{\prime}}$ only. 


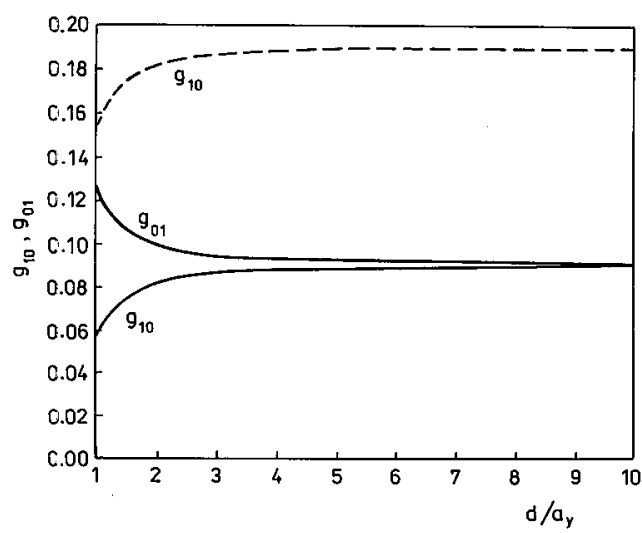

Fig. 1. Dependence of $g_{10}$ and $g_{01}$ on the period $d$ of the lateral multiwire superlattice. The solid lines are for $a_{y}=a_{z}$, and the dashed line is for the 2D model.

Figure 1 shows the variation of $g_{10}$ and $g_{01}$ with the ratio $d / a_{y}$ for LMWSL's with $a_{y}=a_{z}$. We see that the depolarization shift is determined mainly by the intrawire e-e interaction. The interwire interaction decreases very fast with increasing the ratio $d / \alpha_{y}$. This interaction gives substantial contribution to the depolarization shift only in the range $a_{y}<d \leq 2 a_{y}$. Note that the sign of the correction resulting from the interwire interaction is positive for the mode $\tilde{\omega}_{10}$, negative for the mode $\tilde{\omega}_{01}$.

Usually the confinement in the $z$-direction is much stronger thian the confinement in the $y$-direction. So in Fig. 1 we present also the behaviour of $g_{10}$ when $r=a_{z} / a_{y} \rightarrow 0$ (the 2D limit). We find that the contribution to the depolarization shift connected with the intrawire interaction increases with decreasing the ratio $r$, while the contribution connected with the interwire interaction is practically independent of $r$.

\section{References}

[1] T. Egeler, G. Astreiter, G. Weimann, T. Demel, D. Heitmann, P. Grambow, W. Schlapp, Phys. Rev. Lett. 65, 1804 (1990).

[2] J.S. Weiner, G. Danan, A. Pinczuk, J. Valadares, L.N. Pfeiffer, K. West, Phys. Rev. Lett. 63, 1641 (1989).

[3] W. Hansen, M. Horsk, J. P. Kotthaus, U. Merkt, Ch. Sikorski, K. Ploog, Phys. Rev. Lett. 58, 2586 (1987).

[4] T. Demel, D. Heitmann, P. Grambow, K. Ploog, Phys. Rev. B 38, 12732 (1988).

[5] Wei-ming Que, G. Kirczenow, Phys. Rev. B 37, 7153 (1988).

[6] Q.P. Li, S. Das Sarma, Phys. Rev. B 43, 11768 (1991).

[7] H. Ehrenreich, M.H. Cohen, Phys. Rev. 115, 786 (1959). 